\title{
A New Approach of Disaster Forecasting Based on Least Square Optimized Neural Network
}

\author{
Fanbao Meng, ${ }^{1}$ Suolin Jing $\mathbb{D},{ }^{1}$ Xizhen Sun, ${ }^{2}$ Changxiang Wang, ${ }^{1}$ Yanbo Liang, \\ and Da Pang \\ ${ }^{1}$ State Key Laboratory of Mining Disaster Prevention and Control Cofounded by Shandong Province and the Ministry of Science \\ and Technology, Shandong Province (Shandong University of Science and Technology), Qingdao 266590, China \\ ${ }^{2}$ School of Civil Engineering and Architecture, Linyi University, Linyi 276000, China \\ ${ }^{3}$ Weinan Supervisory Branch of Shaanxi Administration of Coal Mine Safety, Shaanxi Province, Weinan 710001, China
}

Correspondence should be addressed to Suolin Jing; 13573246956@163.com

Received 13 August 2020; Accepted 16 October 2020; Published 7 November 2020

Academic Editor: Hualei Zhang

Copyright (C) 2020 Fanbao Meng et al. This is an open access article distributed under the Creative Commons Attribution License, which permits unrestricted use, distribution, and reproduction in any medium, provided the original work is properly cited.

\begin{abstract}
The evaluation of the risk is the prerequisite for the implementation of countermeasures in the prevention and control of rock burst, and the research on the fast forecast at scene of the rock burst is more important for the safety production of coal mine. Aiming at the problem that dynamic disasters caused by many factors and heterogeneity of coal and rock are difficult to predict in the process of coal mining, in this paper, the general law and the risk control factors of the rock burst are studied, a mathematical model based on the BP neural network was built according to the different actual mining conditions in the mining area, and the output layer has obtained the prediction result. Then, the results of the output samples after training were fitted by using SPSS software, and the fitting function was obtained by multiple least square fitting. Finally, the fitting results are checked by the data of actual coal mine dynamic disaster parameters. The prediction results show that the simulation results of BP neural network prediction model and the fitting function of the least square method can reduce the impact of subjective judgment on the prediction results, and the application of the fitting function can obtain the prediction results in the first time to ensure the construction safety. The method of on-site hazard assessment and inspection by using fitting function is simple and feasible and has high accuracy, which provides a new idea for the field prediction of rock burst.
\end{abstract}

\section{Introduction}

Rock burst is one of the common dynamic disasters in coal mine production and becoming more and more serious with the increase of mining depth and intensity year by year [1]. The rock burst is a quite complicated dynamic phenomenon, and it has numerous influence factors; it is the result of comprehensive action of factors such as ground stress and physical and mechanical properties of coal, and the influence factors are highly fuzzy and nonlinear.

At present, a series of theoretical and technical measures which are about risk assessment, prevention, and prediction of rock burst have been developed, including stiffness theory, strength theory, energy theory, "three criteria" theory, catastrophe theory, grey theory, comprehensive index method, catastrophe progression method, analytic hierarchy process, and chaos theory [2-4]. The monitoring methods include electromagnetic radiation [5], acoustic emission observation $[6,7]$, microseismic monitoring [8], tomography technology [9], and drilling cuttings [10]. With the increasingly severe situation of rock burst in coal mines, the higher requirements have been put forward to the monitoring and prediction of rock burst. However, the existing methods are often single in consideration of factors and indicators to solve this nonlinear problem, and there are still existing problems in prediction precision and reliability. Therefore, how to comprehensively consider the interaction and nonlinear relationship of many factors and realize the accurate and efficient on-site prediction of the rock burst has become the key to the prevention and control of the rock burst. 
As a method of nonlinear approximation ability based on black box theory, a neural network has unique advantages in the information mapping party. It can capture the relevant laws between the influence factors and the outstanding events in the dynamic disaster data and combine the qualitative and quantitative. It can also reduce the subjectivity and is scientific and reasonable, the calculation process is simple, the calculation results are accurate, and it has been widely applied in the prediction of rock burst, coal, and gas outburst. The ideal effect can be obtained by using the neural network model; therefore, many experts and scholars at home and abroad carried out a lot of research and produced important results in this field [11-13]. In order to effectively predict and prevent the rock burst occurred in coal mine, Wang et al. [14] improved the BP neural predicted network in combination with the acoustic emission technology and the neural network. A new linear generation mechanism (LGMS) was used by Liu and $\mathrm{Li}[15,16]$ to modify the fruit fly optimization algorithm (FOA) to optimize the generalized regression neural network (GRNN) and BP neural network; a prediction model of rock burst was established which has good prediction and generalization ability. For the purpose of more reasonably and effectively solving the risk prediction problem of rock burst in coal mine, Gao et al. [17] introduced the Principal Component Analysis (PCA) to compress the information from input samples of the GRNN. Li et al. [18] established the evaluation model which is based on Principal Component Analysis and RBF Neural Network. The prediction results indicated that the PCA-GRNN model and PCA-RBF model showed more excellent network performances and higher prediction accuracy and generalization ability. Zhou and Wang [19] developed the probabilistic neural network (PNN) based on Bayesian criteria of multivariate pattern classification. Afraei et al. [20] developed intelligent classification models for rock burst prediction by using five widespread techniques including artificial neural network techniques; different classification models are trained and tested with the same corresponding datasets to evaluate and compare their performances in the similar conditions. The two main components of machine learning [21], artificial neural network (ANN) and support vector machine (SVM), have been used for rock burst prediction. Zhang et al. $[22,23]$ established the prediction model which is based on the SVM theory considering the influencing factors of rock burst. And the statistical learning method, SVM, is employed for kimberlite burst prediction by Yuanyuan et al. [24].

In summary, the analysis process of neural network operation is complicated, and it is generally required to be programmed by a computer. It is difficult for the frontline workers to get the first hand information about the prediction results of rock burst, and in situ accurate and efficient prediction of rock burst needs further study. As a mathematical optimization technique, least squares are used to minimize the quadratic sum of error to find the best function matching of data. The least square method can be used to obtain the unknown data simply and make the quadratic sum of errors between the obtained data and the actual data smallest. The thought of the least square method is used in this paper to fit all the influence factors and the risk index of rock burst and obtains the least square fitting function. After monitoring the factors that affect the risk of rock burst, we can normalize the data and calculate the impact risk with the help of least square fitting function. The combination of artificial neural network and least squares fitting function improves the efficiency of initial prediction and monitoring. At the same time, they mutually verify and effectively improve the accuracy of prediction and explore new ideas for the field prediction and prevention of rock burst.

\section{Design of BP Neural Network Based on Matlab}

An artificial neural network is a new information processing system based on the preliminary understanding of the structure and activity mechanism of the human brain [25]. It is a nonlinear complex network system, which is interconnected with neuron processing units similar to neuron. On the basis of understanding the neural network of the human brain, the neural network of the human brain is abstracted with mathematical and physical methods and information processing angle, and a simplified model is established. It is divided into feedforward network and feedback network according to network structure [26].

The error back propagation neural network is a multilayer feedforward network. The weights are adjusted by the backpropagation learning algorithm; this network is also known as the BP network [27-30]. The learning process consists of forward propagation of information and error backpropagation. The $S$ function is used as the transfer function of its neuron. In the process of forward propagation of information, the input parameters are transferred from the middle layer through the input layer to the output layer, and the arbitrary nonlinear mapping from input to output can be realized. If the output layer does not get the desired output, then the error back propagation is transferred. At this time, the error signal is transmitted from the output layer to the input layer and adjusts the connection weights and thresholds of each layer in time to reduce the error until the target accuracy is reached. At present, the vast majority of neural network applications all adopt the BP network or its improved form, which is the core part of the forward network and embodies the essence of the neural network. The typical BP neural network has five kinds of intelligent characteristics, including associative memory, nonlinear mapping, classification and recognition, optimization calculation, and knowledge processing. The three-level perceptron includes an input layer, hidden layer, and output layer. Information is implied in a network connected by a large number of neurons, without explicit description and the introduction of a nonlinear transfer function, which provides a powerful tool for the processing of nonlinear complex problems. Moreover, the neural network has the ability of self-learning, which can train new data, can adjust the model, and is adaptive to the dynamic process.

The calculation and deduction process of neural network is complex. Matlab is one of the most outstanding software of numerical calculation and simulation analysis. It has many advantages, such as high programming efficiency, strong 
expansion ability, convenient drawing function, simple sentence, rich connotation, and high efficiency in matrix and array. The neural network toolbox developed in the Matlab environment is based on the neural network theory; it can construct the activation function of the typical neural network, so that the designer's calculation of the output of the selected network becomes a call to the activation function. SPSS is a series of software products and related services for IBM, which are used for statistical analysis, data mining, prediction analysis, and decision support tasks. In the "regression" submenu of SPSS, a very rich and powerful regression modelling function is provided. Based on the idea of least squares [31], for the regression model of more than one independent variable, the application of SPSS software can easily get the desired results.

2.1. Construction of BP Neural Network Model. Rock burst is a complicated dynamic phenomenon in mine. Its mechanical environment, location, and macroscopic and microscopic appearance are varied, and the impact strength and damage degree are different. The deformation energy of high strength coal (rock) induced by mine mining can be released instantaneously. It can cause coal and rock mass to be thrown out in the corresponding mining space, causing the damage of the support, the roof fall, the blockage of the roadway, the injured people, the great noise, and rock mass shock, which are all considered to be dangerous to rock burst. The number of nodes in the BP network input layer depends on the dimension of the data source. The number of nodes in the output layer depends on the classification of the research objects. The main factors affecting rock burst are selected as the input parameters of the model. Determining the characteristic index that can reflect the law of rock burst is the premise of establishing the prediction model of rock burst. [32-34] The main factors of rock burst are basically the same according to previous analysis. It can be summarized as the result of the comprehensive effect of the main factors, such as X1 mining depth, X2 coal seam thickness, X3 coal seam dip angle, X4 roof lithology, X5 geological structure, X6 coal rock strength, and $\mathrm{X} 7$ mining means. So the above factors are used as input variables. The quantitative indicators of the input layer are normalized and normalized to the closed area of [0 1$]$. The output variable is whether there is danger of rock burst in monitoring sites. The output layer of the neural network uses 1 and 0 , respectively, to indicate the risk of the rock burst. The weak impact on the working face is relatively small. In order to simplify the analysis, the neural network analysis is unified according to 0 , and the medium or above rock burst is calculated according to 1 [35].

Usually, the number of hidden layers and the number of hidden neurons determine the speed, convergence, and storage space of neural network. The number of nodes in the network has a great influence on its generalization ability. The number of nodes is too few and can not fit the sample data. The number of nodes is too large, then it tends to remember all the learning data, and also includes noise interference, but reduces the generalization performance. According to previous rich theories and practices, a 3-level BP network with a hidden layer can approximate any rational function [36].
Increasing the number of hidden layers can further reduce errors and improve accuracy, but at the same time complicate the network, thus increasing the training time of network weights. In order to reduce the scale and complexity of the system as far as possible, this paper adopts the 3-level improved BP network model with 1 input layer, 1 hidden layer, and 1 output layer. Then the network training is carried out, and the BP artificial neural network model of rock burst prediction is obtained when the network converges. The measured values of sample parameters that need to be predicted for rock burst are taken as input quantities by using this model, and the rock burst grade is determined according to actual output.

The choice of the number of neurons in the hidden layer is a very complex problem, which is caused by the complexity of the network mapping and the uncertainty of the training process. It often needs to be determined according to the experience of the designer and the repeated simulation test. Most methods of selecting hidden nodes are based on an empirical formula. On the basis of empirical formula, the number of nodes is determined according to the predictive effect of neural network. The model correlation is poor when the number of hidden layer nodes is less than 12 and more than 16. The actual prediction ability of the nodes in the 12-16 range is tested, and the 15 layers of hidden layer nodes are selected, and the data simulation of different hidden layer nodes is shown in Table 1.

The convergence factor $\eta$ represents the learning rate, also known as the step length. The number of network training will increase, and the learning efficiency will be slow when the value of $\eta$ is too small, but the learning process is relatively stable. If the value of $\eta$ is larger, the learning process will go on very fast, but the learning process is too fast; it may cause the instability of the solution process, but will lead to the increase of the error $E$. The maximum training frequency is $1 e 3$, and the training accuracy is $1 e-3$. The $\mathrm{BP}$ algorithm is used to train the network. When training target is met or the maximum number of iterations is satisfied, training is stopped automatically. The built network structure model is shown in Figure 1.

In Matlab, the "newff" function can be used to create the BP neural network, which requires four input conditions: the maximum and minimum of the input samples of the $R$ dimension, the number of neurons in each layer, the transfer function of each layer of neurons, and the name of the training function. After creating the BP network, the network automatically initializes the weights and thresholds, with a default value of 0 . In this paper, the weights and thresholds of the network determined by the actual size of the impact are determined. The $S$ function is used to input the given network structure and input matrix, and the result of simulation operation is obtained. Matlab provides a variety of training functions for different conditions, and the weights and thresholds of the network will be adjusted repeatedly to reduce the default value of the performance function.

2.2. Application of BP Neural Network Model. Based on the actual situation of the adjacent mining areas of a rock burst mine in Shandong Province, China, the rock burst situation 
TABLE 1: Data simulation of different hidden layer neuron number.

\begin{tabular}{|c|c|c|c|c|c|c|}
\hline \multirow{2}{*}{$\begin{array}{l}\text { Training } \\
\text { data }\end{array}$} & \multicolumn{5}{|c|}{ Prediction results of different hidden layers } & \multirow{2}{*}{$\begin{array}{l}\text { Actual } \\
\text { results }\end{array}$} \\
\hline & 12 & 13 & 14 & 15 & 16 & \\
\hline Name A & 0.907 & 0.872 & 0.915 & 0.957 & 0.960 & 1 \\
\hline Name B & 0.644 & 0.529 & 0.514 & 0.506 & 0.704 & 0 \\
\hline Name C & 0.811 & 0.971 & 0.869 & 0.967 & 0.986 & 1 \\
\hline Name D & 0.526 & 0.521 & 0.507 & 0.503 & 0.561 & 0 \\
\hline Name E & 0.960 & 0.948 & 0.905 & 0.917 & 0.914 & 1 \\
\hline Name F & 0.946 & 0.896 & 0.880 & 0.948 & 0.927 & 1 \\
\hline Name G & 0.533 & 0.556 & 0.509 & 0.522 & 0.535 & 0 \\
\hline Name H & 0.898 & 0.903 & 0.928 & 0.954 & 0.958 & 1 \\
\hline Name I & 0.503 & 0.579 & 0.689 & 0.518 & 0.502 & 0 \\
\hline Name J & 0.960 & 0.921 & 0.939 & 0.945 & 0.940 & 1 \\
\hline
\end{tabular}

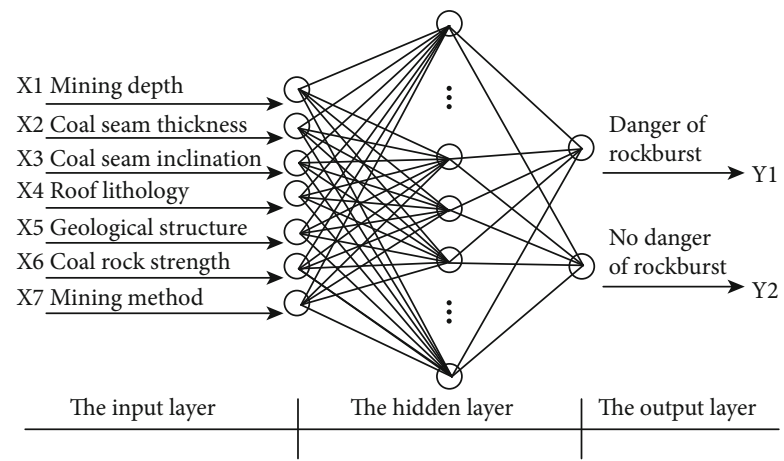

FIgURE 1: Schematic diagram of BP network structure.

at different locations near different mining areas was studied. In order to achieve the effect of fast and accurate on-site forecast, all parameters are quantified by on-site technicians. In order to meet the requirement of BP neural network, avoid the problem of convergence caused by small signal data submergence and singular data. At the same time, in order to speed up the operation, all data are normalized. Meanwhile, in order to improve the reliability of the data, the quantification process is carried out by a unified standard. Mining depth, coal seam thickness, and coal seam dip are normalized by a formula based on measured results.

$$
y=\frac{(x-\text { MinValue })}{(\text { MaxValue }- \text { MinValue })} .
$$

In the formula, $x$ and $y$ are the values before and after conversion and MaxValue and MinValue are the maximum and minimum values of samples, respectively.

The parameters of roof management, geological structure, strength of coal rock, and mining method are classified into four grades according to the simple, general, medium, to complex tendency of disaster induction and expressed by 0.1 , $0.4,0.7$, and 1.0. The corresponding normalized description is shown in Table 2 . The first 20 sets of data are used as training data, and the latter 10 groups are taken as experimental data for analysis.

\section{Least Square Estimation of the Fitting Coefficient Based on SPSS}

In the research of statistical methods of scientific experiments, it is necessary to find out the relationship between variables and results from a set of experimental data $(X i, Y i$ ), where $i$ is a positive integer between 0 and $M$. Since the observational data often have errors, we do not ask $y=F(x)$ to pass all of the points $(X i, Y i)$, and only require that the error on a given $X i$ be minimized by a certain standard. Therefore, in the actual field of coal mine, after measuring the key variables affecting the rock burst, the data is fitted by numerical analysis. Using this idea and combining the advantages of numerical analysis, the risk of the rock burst can be further divided more carefully, and the risk of impact is divided into four grades from micro impact, weak impact, general impact, and high impact risk. The results are expressed in $0.1,0.4,0.7$ and 1 , respectively, and the data are fitted. The results after fitting were analyzed. If verified by the actual geological conditions, the impact risk can be given in time when the danger signs are not significant; it can provide safety and guarantee for the first-line production of coal mines.

The measured values of the main influencing factors and a set of weight coefficients are known, requiring function:

$$
S_{n}=\left(x_{1}, x_{2}, x_{3}, \cdots, x_{i}\right)=\sum_{k=1}^{n} a_{k} \varphi_{k}\left(x_{1}, x_{2}, x_{3}, \cdots, x_{i}\right), \quad n \leq m .
$$

It makes that

$$
F\left(a_{0}, a_{1}, \cdots, a_{n}\right)=\sum_{i=1}^{m} \omega_{i}\left[y_{i}-S_{n}\left(x_{1}, x_{2}, x_{3}, \cdots, x_{i}\right)\right]^{2} .
$$

The following are the satisfied normal equations:

$$
\sum_{j=0}^{n}\left(\varphi_{k}, \varphi_{j}\right) a_{j}=d_{k} \quad(k=0,1, \cdots, n)
$$

$$
\left(\varphi_{k}, \varphi_{j}\right)=\sum_{i=1}^{m} \omega_{i} \varphi_{k}\left(x_{1}, x_{2}, x_{3}, \cdots, x_{i}\right) \varphi_{i}\left(x_{1}, x_{2}, x_{3}, \cdots, x_{i}\right) \text {. }
$$

The coefficient is obtained, and the least square fitting is obtained.

Because of the large amount of data, the traditional manual algorithm is slow and the accuracy is not high. Based on the thought of the least square method, using the SPSS software, the least square fitting can be used quickly to get all the results of the coefficient and the correlation test. The function expressions obtained by using the above method are as follows:

$$
\begin{aligned}
S_{n}(x)= & 0.345 * x_{1}-0.045 * x_{2}+0.167 * x_{3}+0.154 \\
& * x_{4}-0.117 * x_{5}+0.149 * x_{6}+0.505 * x_{7} .
\end{aligned}
$$

The fitting reaches 0.99 , which meets the requirement of accuracy and has a good correlation. It can be applied to the 
TABLE 2: Sample quantization table.

\begin{tabular}{|c|c|c|c|c|c|c|c|c|}
\hline Name & Mining depth & Mining thickness & Coal seam dip & $\begin{array}{c}\text { Roof } \\
\text { management }\end{array}$ & $\begin{array}{l}\text { Geological } \\
\text { structure }\end{array}$ & $\begin{array}{l}\text { Strength of coal } \\
\text { and rock }\end{array}$ & Mining method & Impact situation \\
\hline 1 & 0.45 & 0.50 & 0.83 & 0.10 & 0.40 & 0.40 & 0.10 & Weak impact \\
\hline 2 & 0.36 & 0.18 & 0.15 & 0.40 & 0.10 & 0.40 & 0.70 & General impact \\
\hline 3 & 1.00 & 1.00 & 0.74 & 0.40 & 1.00 & 0.70 & 0.40 & General impact \\
\hline 4 & 0.65 & 0.23 & 0.51 & 0.40 & 0.70 & 0.70 & 0.10 & Weak impact \\
\hline 5 & 0.70 & 0.73 & 0.47 & 0.70 & 0.70 & 0.70 & 0.70 & General impact \\
\hline 6 & 0.04 & 0.36 & 0.89 & 0.10 & 1.00 & 0.70 & 0.10 & Micro impact \\
\hline 7 & 0.48 & 0.27 & 0.51 & 0.70 & 0.10 & 0.40 & 1.00 & High impact \\
\hline 8 & 0.82 & 0.82 & 0.60 & 0.10 & 0.70 & 0.40 & 0.10 & Weak impact \\
\hline 9 & 0.43 & 0.82 & 0.94 & 1.00 & 1.00 & 1.00 & 1.00 & High impact \\
\hline 10 & 0.43 & 0.32 & 0.32 & 1.00 & 0.10 & 0.10 & 0.10 & Weak impact \\
\hline 11 & 0.34 & 0.27 & 0.32 & 0.70 & 0.10 & 0.40 & 0.70 & General impact \\
\hline 12 & 0.47 & 0.00 & 0.30 & 0.70 & 0.40 & 0.40 & 0.10 & Weak impact \\
\hline 13 & 0.58 & 0.55 & 0.32 & 0.40 & 0.40 & 0.70 & 0.70 & General impact \\
\hline 14 & 0.52 & 0.36 & 0.32 & 0.40 & 0.70 & 0.40 & 1.00 & General impact \\
\hline 15 & 0.38 & 0.59 & 1.00 & 0.10 & 1.00 & 0.70 & 0.40 & Weak impact \\
\hline 16 & 0.63 & 0.64 & 0.38 & 0.70 & 0.70 & 0.70 & 0.70 & General impact \\
\hline 17 & 0.40 & 0.36 & 0.43 & 0.40 & 1.00 & 0.10 & 0.70 & Weak impact \\
\hline 18 & 0.51 & 1.00 & 0.09 & 0.10 & 0.70 & 0.40 & 0.10 & Micro impact \\
\hline 19 & 0.38 & 0.18 & 0.15 & 0.40 & 0.10 & 0.10 & 0.40 & Weak impact \\
\hline 20 & 0.26 & 0.18 & 0.17 & 0.40 & 0.10 & 0.40 & 0.70 & Weak impact \\
\hline 21 & 0.50 & 0.32 & 0.96 & 0.70 & 0.40 & 0.70 & 0.40 & General impact \\
\hline 22 & 0.47 & 0.18 & 0.43 & 0.70 & 0.70 & 0.40 & 0.40 & Weak impact \\
\hline 23 & 0.34 & 1.00 & 0.30 & 1.00 & 0.70 & 0.40 & 1.00 & General impact \\
\hline 24 & 0.49 & 0.09 & 0.00 & 0.10 & 0.10 & 0.10 & 0.10 & Micro impact \\
\hline 25 & 0.42 & 0.27 & 0.34 & 0.70 & 0.40 & 0.70 & 0.70 & General impact \\
\hline 26 & 0.32 & 2.36 & 0.21 & 0.70 & 0.10 & 0.70 & 1.00 & General impact \\
\hline 27 & 0.00 & 0.45 & 0.89 & 0.10 & 1.00 & 0.40 & 0.40 & Micro impact \\
\hline 28 & 0.81 & 0.82 & 0.53 & 0.70 & 0.70 & 1.00 & 1.00 & High impact \\
\hline 29 & 0.53 & 0.18 & 0.38 & 0.10 & 0.40 & 0.10 & 0.10 & Micro impact \\
\hline 30 & 0.77 & 0.45 & 0.53 & 0.40 & 0.70 & 0.40 & 0.70 & General impact \\
\hline
\end{tabular}

prevention and preliminary prediction of rock burst in mines.

\section{Comparison of Forecast Results and Actual Situation}

According to the sample quantization table above, the least square fitting is done by using Matlab, and the fitting result is obtained. The results of the least squares fitting and actual situation are shown in Figure 2.

Through the analysis of the prediction results, it can be clearly seen that the application of the fitting function can accurately predict the outburst risk of the locations at the first time, which avoids the influence of humanistic subjective factors to a certain extent. If the fitting result is more than 0.5 , it indicates that there is a large impact tendency under this condition.

The combined method of artificial neural network and least square method is used to study the prediction of high impact ground pressure in mining area. The practical

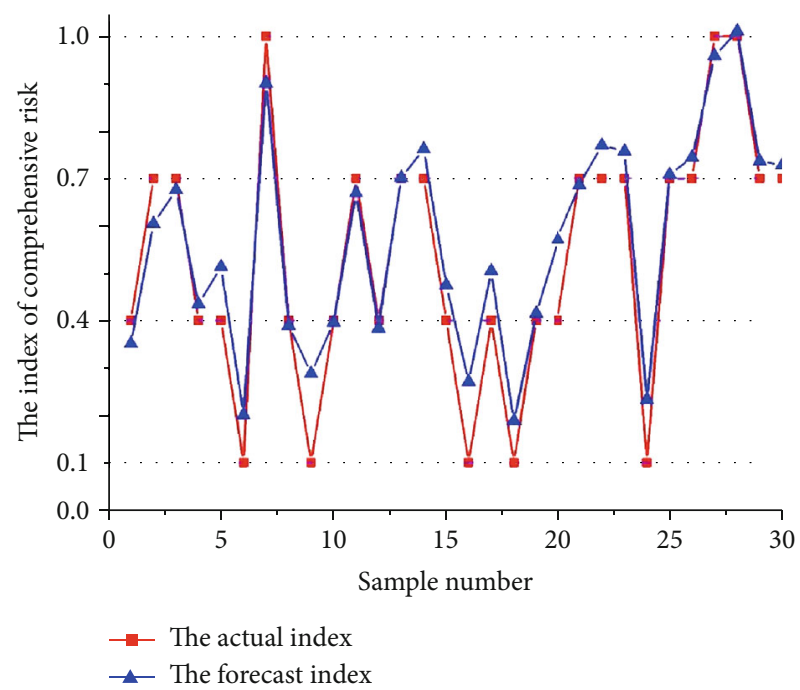

Figure 2: Comparison of forecast results and actual situation. 
application shows that the prediction results of this method are in good agreement with the actual situation, and it is an effective method for predicting the rock burst. In combination with the two methods, if the risk of impact is strong, it is necessary to take the next step of unloading and reducing the disaster in time to prevent the occurrence of the accident. In summary, it is feasible to use fitting function and neural network to prevent and monitor the danger of rock burst.

\section{Conclusion}

(1) In view of the current situation of the increasing impact of rock burst disaster, in order to accurately and conveniently carry out the fast prediction and prediction of the rock burst, the neural network and the least square fitting ideas are used, and a risk assessment method based on combination of neural network and least squares fitting function is proposed in this paper

(2) According to the different actual conditions of mining in the mining area, a Matlab mathematical model for risk prediction of rock burst is constructed based on the BP neural network, and the output layer has obtained the prediction result of rock burst danger. The fitting function is obtained by multiple least squares fitting by SPSS software, that is, $S_{n}(x)$

(3) Based on the actual situation of the adjacent mining areas of a mining group, the rock burst situation at different locations near different mining areas was studied and the fitting results were tested. The simulation results of the $\mathrm{BP}$ neural network prediction model and the least square fitting function predict the danger of the rock burst. The results show that the test method is simple and feasible and has high accuracy

\section{Data Availability}

The data used to support the findings of this study are available from the corresponding author upon request.

\section{Conflicts of Interest}

The authors declare no conflicts of interest.

\section{Acknowledgments}

This study was supported by the Shandong Provincial Natural Science Foundation of China (ZR2019BEE013) and the National Natural Science Foundation of China (Nos. 51974174 and 51974173).

\section{References}

[1] X. Wang and F. B. Meng, "Statistical analysis of large accidents in China's coal mines in 2016," Natural Hazards, vol. 92, no. 1, pp. 311-325, 2018.
[2] C. Wang, A. Wu, H. Lu, T. Bao, and X. Liu, "Predicting rockburst tendency based on fuzzy matter-element model," International Journal of Rock Mechanics and Mining Sciences, vol. 75, pp. 224-232, 2015.

[3] Q. Yu, C. A. Tang, L. Li, G. W. Cheng, and L. X. Tang, "Study on rockburst nucleation process of deep-buried tunnels based on microseismic monitoring," Shock and Vibration, vol. 2015, Article ID 685437, 17 pages, 2015.

[4] Z. J. Wen, S. L. Jing, Y. J. Jiang et al., "Study of the Fracture Law of Overlying Strata under Water Based on the Flow-StressDamage Model," Geofluids, vol. 2019, Article ID 3161852, 12 pages, 2019.

[5] V. Frid, "Rockburst hazard forecast by electromagnetic radiation excited by rock fracture," Rock Mechanics and Rock Engineering, vol. 30, no. 4, pp. 229-236, 1997.

[6] T. H. W. Goebel, T. W. Becker, D. Schorlemmer et al., "Identifying fault heterogeneity through mapping spatial anomalies in acoustic emission statistics," Journal of Geophysical Research: Solid Earth, vol. 117, no. B3, 2012.

[7] S. A. Hall, F. D. Sanctis, and G. Viggiani, "Monitoring fracture propagation in a soft rock (neapolitan tuff) using acoustic emissions and digital images," Pure and Applied Geophysics, vol. 163, no. 10, pp. 2171-2204, 2006.

[8] C. Tang, L. Li, N. Xu, and K. Ma, "Microseismic monitoring and numerical simulation on the stability of high-steep rock slopes in hydropower engineering," Journal of Rock Mechanics and Geotechnical Engineering, vol. 7, no. 5, pp. 493-508, 2015.

[9] N. Hosseini, "Evaluation of the rockburst potential in longwall coal mining using passive seismic velocity tomography and image subtraction technique," Journal of Seismology, vol. 21, no. 5, pp. 1101-1110, 2017.

[10] G. Tokar, "Model trials for test drilling to anticipate rockburst hazard (In German)," International Journal of Rock Mechanics and Mining Sciences \& Geomechanics Abstracts, vol. 26, no. 2, pp. 77-77, 1989.

[11] R. Trivedi, T. N. Singh, and A. K. Raina, "Prediction of blastinduced flyrock in indian limestone mines using neural networks," Journal of Rock Mechanics and Geotechnical Engineering, vol. 6, no. 5, pp. 447-454, 2014.

[12] A. C. Adoko, C. Gokceoglu, L. Wu, and Q. J. Zuo, "Knowledgebased and data-driven fuzzy modeling for rockburst prediction," International Journal of Rock Mechanics and Mining Sciences, vol. 61, no. 4, pp. 86-95, 2013.

[13] H. Guo, M. Ji, K. Chen, Z. Zhang, Y. Zhang, and M. Zhang, "The feasibility of mining under a water body based on a fuzzy neural network," Mine Water and the Environment, vol. 37, no. 4, pp. 703-712, 2018.

[14] Y. H. Wang, L. L. Liu, H. Fu, and Y. S. Xu, "Study on predicted method of mine pressure bump based on improved BP neural network," Coal Science and Technology, vol. 45, no. 10, pp. 3640, 2017.

[15] X. Y. Liu and P. Y. Li, "Prediction of rock burst based on improved FOA-BP neural network," Mining safety \& environmental protection, vol. 45, no. 3, pp. 55-60, 2018.

[16] X. Y. Liu, P. Y. Li, and Y. J. Zhou, "Research on the prediction of rock burst based on generalized regression neural network optimized by improved Drosophila algorithm," Mining Research \& Development, vol. 37, no. 10, pp. 106-110, 2017.

[17] F. Gao, L. C. Chen, and L. G. Wang, "Neural network method of PCA and GRNN for rock burst prediction in coal mine," China Coal, vol. 42, no. 4, pp. 48-52, 2016. 
[18] X. B. Li, W. Zhu, W. J. Liu, and D. M. Zhang, "Research on rock mass blastability based on principal component analysis and RBF neural network," Gold Science and Technology, vol. 23, no. 6, pp. 58-63, 2015.

[19] Y. Zhou and T. Wang, "PNN-based rockburst prediction model and its applications," Earth Sciences Research Journal, vol. 21, no. 3, pp. 141-146, 2017.

[20] S. Afraei, K. Shahriar, and S. H. Madani, "Developing intelligent classification models for rock burst prediction after recognizing significant predictor variables, section 2: designing classifiers," Tunnelling and Underground Space Technology, vol. 84, pp. 522-537, 2019.

[21] P. C. Jackson, Introduction to Artificial Intelligence, Courier Dover Publications, 2019.

[22] M. Zhang, J. H. Chen, and Z. Y. Zhou, "Grading prediction model of rock burst based on SVM and its R language description," The Chinese Journal of Geological Hazard and Control, vol. 29, no. 4, pp. 64-69, 2018.

[23] M. Y. Qiao, P. F. Cheng, Z. Z. Liu, and Y. X. Liu, "Prediction of rock burst risk grade based on improved FOA-SVM," The Chinese Journal of Geological Hazard and Control, vol. 29, no. 4, pp. 70-77, 2018.

[24] P. Yuanyuan, D. B. Apel, W. Chao, and W. Brandon, "Evaluation of burst liability in kimberlite using support vector machine," Acta Geophysica, vol. 66, pp. 973-982, 2018.

[25] A. M. Remennikov and P. A. Mendis, "Prediction of airblast loads in complex environments using artificial neural networks," Structures under Shock and Impact, vol. 87, pp. 269278, 2006.

[26] R. Haoyuan, Research on application of BP neural network in coal and gas outburst, Doctoral dissertation, Xi'an University of Science and Technology, 2012.

[27] D. E. Rumerlhar, "Learning representation by backpropagating errors," Nature, vol. 323, no. 3, pp. 533-536, 1986.

[28] J. Cheng, X. Wang, T. Si et al., "Maximum burning rate and fixed carbon burnout efficiency of power coal blends predicted with back-propagation neural network models," Fuel, vol. 172, pp. 170-177, 2016.

[29] M. Khandelwal and T. N. Singh, "Prediction of macerals contents of Indian coals from proximate and ultimate analyses using artificial neural networks," Fuel, vol. 89, no. 5, pp. 1101-1109, 2010.

[30] S. C. Chelgani, J. C. Hower, E. Jorjani, S. Mesroghli, and A. H. Bagherieh, "Prediction of coal grindability based on petrography, proximate and ultimate analysis using multiple regression and artificial neural network models," Fuel Processing Technology, vol. 89, no. 1, pp. 13-20, 2008.

[31] O. Stan and E. Kamen, "A local linearized least squares algorithm for training feedforward neural networks," IEEE Transactions on Neural Networks, vol. 11, no. 2, pp. 487-495, 2000.

[32] H. Yi and S. Wanstedt, "The introduction of neural network system and its applications in rock engineering," Engineering Geology, vol. 49, no. 3, pp. 1093-1093, 1998.

[33] Z. X. Zhang, "Rock mechanics related to mining engineering," in ISRM 3rd Nordic Rock Mechanics Symposium - NRMS 2017, Helsinki, Finland, October 2017.

[34] K. Yang and J. H. Chen, "Research of rock burst prediction based on combination of principal component analysis and neural network," Journal of Guangxi University, vol. 37, no. 5, pp. 997-1003, 2012.
[35] Y. S. Li, K. Ai, and J. M. Yin, "Application of bp network based on vb and Matlab in analysis of in-situ stress field," Journal of Yangtze River Scientific Research Institute, vol. 26, no. 6, pp. 24-27, 2009.

[36] W. Wang and Y. Zhang, "Artificial neural networks for predicting rockburst in deep mining," Journal of Hebei Normal University of Science \& Technology, vol. 2, 2007. 